\title{
Perfil clínico y etiológico de pacientes operados con endocarditis activa. Seguimiento a diez años
}

\author{
Dres. Diego Pérez Zerpa, Amparo Fernández, Facundo Ríos, Eloísa Silva, \\ Macarena Lorente, María José Arocena, Jorge Estigarribia, Gerardo Soca, \\ Víctor Dayan
}

\begin{abstract}
Resumen ticas clínicas y evolutivas de pacientes operados por endocarditis infecciosa activa. tivos enunciados. cedimientos invasivos como causas probables.

Palabras clave: $\quad$ ENDOCARDITIS

EMBOLIAS

COMPLICACIONES LOCALES

COMPLICACIONES PERIVALVULARES

COMPLICACIONES PARAVALVULARES

ENDOCARDITIS PROTÉSICA

STAPHYLOCOCCUS AUREUS
\end{abstract}

Antecedentes: la endocarditis infecciosa es una enfermedad con elevada morbimortalidad, la cual se encuentra en torno al 25\%. Aproximadamente el 50\% de los casos requiere tratamiento quirúrgico. En nuestro medio se desconocen las caracterís-

Objetivos: primario, determinar mortalidad operatoria (MO) y sobrevida a largo plazo; secundario, definir predictores de embolias, complicaciones locales (CL), MO y endocarditis protésica (EP) en la evolución.

Métodos: trabajo retrospectivo y analítico. Se identificaron pacientes que recibieron cirugía cardíaca por endocarditis activa entre enero de 2006 y diciembre de 2017. Mediante regresión logística multivariada se identificaron predictores para los obje-

Resultados: se incluyeron 101 pacientes. El microorganismo más frecuentemente encontrado fue Staphylococcus aureus (SA) (15,8\%). La topografía fue protésica en 20,8\%, aórtica en 46,5\%, mitral en 23,8\% y mitro-aórtica en 13,9\%. La MO fue $11,3 \%$ y $29,5 \%$ ( $\mathrm{p}=0,025)$, según ausencia o presencia de CL, único predictor independiente de mortalidad (OR=3,38). El $47,5 \%$ presentó CL, siendo la más frecuente el absceso (25,7\%). Fueron predictores independientes: $\mathrm{EP}(\mathrm{OR}=5,8)$, endocarditis valvular aórtica $(\mathrm{OR}=2,9)$ y sexo masculino $(\mathrm{OR}=3,5)$. La incidencia de $\mathrm{EP}$ precoz fue $3 \%$ y tardía $4 \%$. El $30 \%$ de los pacientes adquirió la EI como consecuencia de un procedimiento médico invasivo en los 6 meses previos. De los siete pacientes con EP en la evolución, seis tenían CL (p<0,05). El 31,7\% presentaba embolias, resultando predictores independientes: Staphylococcus aureus $(\mathrm{OR}=4,6)$, vegetación en el velo mitral posterior $(\mathrm{OR}=3,2)$ y antecedente de hipertensión arterial $(\mathrm{OR}=3,32)$. La sobrevida a cinco y diez años fue de $88,20 \% \pm 0,04$ y $81,50 \% \pm 0,05$ respectivamente.

Conclusiones: la MO de la endocarditis activa en nuestro medio es similar a la reportada internacionalmente. La presencia de CL se asocia a EP en la evolución y resultó ser un predictor independiente de sobrevida a largo plazo, y de MO. La sobrevida a largo plazo es similar a la reportada internacionalmente. Se observó una alta incidencia de agentes intrahospitalarios y pro-

\section{Clinical and etiological profile of patients operated on with active endocarditis. Follow-up at 10 years}

\section{Summary}

Introduction: infective endocarditis is a high morbidity and mortality disease, which is about $25 \%$. About fifty percent of patients require heart surgery. In our environment, clinical and evolutionary characteristics of patients operated with active endocarditis are unknown.

Objective: primary, determine operative mortality and long-term survival; secondary, define predictors of embolisms,

Instituto Nacional de Cirugía Cardíaca. Montevideo, Uruguay.

Correspondencia: Dr. Diego Álvaro Pérez Zerpa. Ejido 1029/201. Montevideo, Uruguay.

Correo electrónico: dperezzerpa@gmail.com

Los autores declaran no tener conflictos de intereses.

El presente estudio no tiene fuentes de financiamiento.

Recibido Nov 11, 2019; aceptado Abr 15, 2020. 
local complications, operative mortality and prosthetic endocarditis in evolution.

Methods: retrospective, analytical study. Patients who received cardiac surgery for active endocarditis between January 2006 and December 2017 were identified. Through multivariate logistic regression, predictors were identified for the stated objectives.

Results: one hundred and one patients were included. The most frequently found microorganism was Staphylococcus aureus (15.8\%). The topography was prosthetic in $20.8 \%$, aortic $46.5 \%$, mitral $23.8 \%$ and mitro-aortic $13.9 \%$. The operative mortality was $11.3 \%$ and $29.5 \%(\mathrm{p}=0.025)$ according to the absence or presence of local complications, the only independent predictor of mortality ( $\mathrm{OR}=3.32)$. Local complications were present in $47.5 \%$, the most frequent were abscess $(25.7 \%)$, independent predictors were: prosthetic endocarditis $(\mathrm{OR}=5.8)$, aortic endocarditis $(\mathrm{OR}=2.9)$ and male sex $(\mathrm{OR}=3.5)$. The incidence of early prosthetic endocarditis was $3 \%$ and late $4 \%$. Thirty percent of patients acquired infective endocarditis as a result of an invasive medical procedure in the previous 6 months. Of the seven patients with prosthetic endocarditis in evolution, six had local complications $(\mathrm{p}<0.05)$. Embolic events were present in $31.7 \%$ of patients, were independent predictors: Staphylococcus aureus $(\mathrm{OR}=4.6)$, presence of vegetation in the posterior mitral leaflet $(\mathrm{OR}=3.2)$ and history of hypertension (OR=3.32). Survival at 5 and 10 years was $88.20 \% \pm 0.04$ and $81.50 \% \pm 0.05$ respectively.

Conclusions: operative mortality of active endocarditis in our environment is high and similar to that reported internationally. The presence of local complications is associated with prosthetic endocarditis in evolution and proved to be an independent predictor of long-term survival, and operative mortality. Long-term survival is similar to that reported internationally. A high incidence of in-hospital agents was observed and invasive procedures as probable causes.

Key words:

\author{
ENDOCARDITIS \\ EMBOLISMS \\ LOCAL COMPLICATIONS \\ PERIVALVULAR COMPLICATIONS \\ PARAVALVULAR COMPLICATIONS \\ PROSTHETIC ENDOCARDITIS \\ STAPHYLOCOCCUS AUREUS
}

\title{
Perfil clínico e etiológico dos pacientes operados com endocardite ativa. Acompanhamento aos 10 anos
}

\section{Resumo}

Antecedentes: a endocardite infecciosa é uma doença com alta morbimortalidade. Requerem tratamento cirúrgico o $50 \%$. Em nosso meio, as características clínicas e evolutivas dos pacientes operados com endocardite ativa são desconhecidas.

Objetivos: primário, determinar a mortalidade operatória e a sobrevida a longo prazo; secundário: Definir preditores de embolias, complicações locais, mortalidade operatória e endocardite protética na evolução.

Métodos: trabalho retrospectivo, analítico. Foram identificados no banco de dados pacientes submetidos à cirurgia de endocardite ativa entre janeiro de 2006 e dezembro de 2017. Através de regressão logística multivariada, os preditores foram identificados para os objetivos estabelecidos.

Resultados: cento e um pacientes foram incluídos. Staphylococcus aureus foi o microrganismo mais frequente (15,8\%). A topografia foi protética em 20,8\%, aórtica 46,5\%, mitral 23,8\% e mitroaórtica 13,9\%. A mortalidade operatória foi de 11,3\% e 29,5\% ( $p=0,025$ ), de acordo com a ausência ou presença de complicações locais, o único preditor independente de mortalidade (OR). Um 47,5\% apresentaram complicações locais, sendo o mais frequente o abscesso $(25,7 \%)$. Os preditores independentes foram: endocardite protética $(\mathrm{OR}=5,8)$, endocardite valvar aórtica $(\mathrm{OR}=2,9))$ e sexo masculino $(\mathrm{OR}=3,5)$. A incidência de endocardite protética precoce foi de $3 \%$ e tardia de $4 \%$. Trinta por cento dos pacientes adquiriram endocardite infecciosa como resultado de um procedimento médico invasivo nos 6 meses anteriores. Dos pacientes com endocardite protética na evolução, 85,7\% apresentava complicações locais ( $\mathrm{p}<0,05)$. O 31,7\% apresentava embolia, resultando em preditores independentes: Staphylococcus aureus $(\mathrm{OR}=4,6)$, vegetação no véu mitral posterior $(\mathrm{OR}=3,2)$ e história de hipertensão arterial $(\mathrm{OR}=3,32)$. A sobrevida em 5 e 10 anos foi de 88,20\% $\pm 0,04$ e 81,50\% $\pm 0,05$, respectivamente.

Conclusóes: a mortalidade operatória da endocardite ativa em nosso ambiente é alto e semelhante ao relatado internacionalmente. A presença de complicações locais está associada à endocardite protética na evolução e provou ser um preditor independente de sobrevida a longo prazo e mortalidade operatória. A sobrevivência a longo prazo é semelhante à relatada internacionalmente. Foi observada alta incidência de agentes hospitalares e procedimentos invasivos como causas prováveis.

$\begin{array}{ll}\text { Palavras chave: } & \text { ENDOCARDITE } \\ & \text { EMBOLIAS } \\ & \text { COMPLICAÇÕES LOCAIS } \\ & \text { COMPLICAÇÕES PERIVALVULARES } \\ & \text { COMPLICAÇÕES PARAVALVULARES } \\ \text { ENDOCARDITE PROTÉTICA } \\ \text { STAPHYLOCOCCUS AUREUS }\end{array}$




\section{Introducción}

La endocarditis infecciosa (EI) es una enfermedad poco frecuente, cuya incidencia anual varía entre 3-7/100.000 personas en estudios contemporá neos ${ }^{(1-3)}$. Sigue caracterizándose por una gran morbimortalidad que no ha sido modificada en las últimas cuatro décadas. La mortalidad hospitalaria se encuentra en torno a $25 \%$ (pudiendo ser mayor según el microorganismo involucrado), con una mortalidad al año de hasta $40 \%{ }^{(3,4)}$. En nuestra región, la mortalidad hospitalaria también es elevada, hasta 25,5\% en el registro EIRA 3 (Endocarditis Infecciosa en la República Argentina), publicado en $2018^{(5)}$. Aunque la incidencia ha permanecido estable ${ }^{(1,2,8)}$, el cuadro clínico y el perfil epidemiológico de la EI han cambiado en los últimos 50 años ${ }^{(9,10)}$.

El registro EIRA, que analizó el perfil epidemiológico de la EI en Argentina, constituyó el primer estudio multicéntrico efectuado en nuestra región. Los autores describen características clínicas, terapéuticas y la morbimortalidad de la enfermedad a principios de la década de $1990^{(6)}$. Posteriormente, en 2002 y 2017, se realizaron dos nuevos relevamientos (EIRA 2 y 3$)^{(5,7)}$ que permitieron comparar las características de la EI a lo largo del tiempo, y mostraron importantes diferencias epidemiológicas en coincidencia con las observadas en países del mundo desarrollado. A pesar de grandes avances en tecnología, conocimiento de la enfermedad, métodos diagnósticos precisos y antibióticos (ATB) más eficaces, la mortalidad permanece incambiada.

En nuestro medio se desconocen las características clínicas y evolutivas de los adultos con EI que requieren intervención quirúrgica. Nuestro objetivo primario fue determinar la mortalidad operatoria (MO) y la sobrevida a largo plazo. Nuestro objetivo secundario fue definir predictores de embolias, complicaciones locales (CL), MO y endocarditis protésica (EP) en la evolución.

\section{Método}

Se realizó un trabajo retrospectivo, observacional, aprobado por el Comité de Investigación del Instituto Nacional de Cirugía Cardíaca. Se incluyeron todos los pacientes intervenidos quirúrgicamente por endocarditis activa (EA), definida como aquella bajo tratamiento ATB. La MO fue definida como la muerte ocurrida durante la internación o durante los primeros 30 días de la intervención quirúrgica. Se consideró CL a la presencia (en estudio ecocardiográfico o como hallazgo quirúrgico) de absceso, pseudoaneurisma, fístula o destrucción del aparato $\operatorname{valvular}^{(11)}$.

\section{Principales aportes}

- La mortalidad operatoria y la sobrevida a largo plazo de la población con endocarditis activa que recibió cirugía cardíaca en nuestro medio, son similares a las reportadas internacionalmente.

- Se observó una alta incidencia de agentes intrahospitalarios y procedimientos invasivos como causas probables.

- La presencia de vegetaciones sobre el velo posterior mitral y la infección por Staphylococcus aureus fueron predictores independientes de embolias.

- Los pacientes con complicaciones locales deben ser seguidos estrechamente, incluso a largo plazo, ya que estas resultaron ser el principal predictor de muerte en el seguimiento.

Los pacientes que recibieron cirugía por EA entre enero de 2006 y diciembre de 2017 , se identificaron en la base de datos institucional de recolección prospectiva. Se extrajeron variables demográficas, etiológicas, quirúrgicas, ecocardiográficas y evolutivas. Las variables continuas se expresan como media y desvío estándar, las categóricas como porcentaje. Las comparaciones se realizaron mediante test de t o chi cuadrado, según correspondiera. Mediante regresión logística multivariada se identificaron predictores para los objetivos enunciados. Para el análisis multivariado se consideraron variables con $\mathrm{p}<0,1$ en el univariado. La sobrevida a largo plazo se analizó mediante Kaplan-Meier y se comparó mediante log-rank test. Se determinaron predictores de sobrevida mediante regresión de Cox. Se consideró significativa una $\mathrm{p}<0,05$. Se consideraron de emergencia las intervenciones quirúrgicas realizadas dentro de las 24 horas del diagnóstico, de urgencia las realizadas entre las 24 y las 48 horas y de coordinación las realizadas luego de las 48 horas.

\section{Resultados}

Entre enero de 2006 y diciembre de 2017 se intervinieron quirúrgicamente 101 pacientes con EI definida por criterios de Duke o criterios de Duke modificados, según la fecha de presentación. En la tabla 1 de describen las características clínicas. El microorganismo más frecuentemente identificado fue Staphylococcus aureus (SA) (15,8\%), seguido de enterococo $(14,9 \%)$; la incidencia de los diferentes microorganimos se encuentra en la figura 1. La mayoría de los hemocultivos (HC) fueron positivos $(72,3 \%)$, pero el cultivo valvular fue positivo en una pequeña proporción de casos $(16,8 \%)$ (figura 2$)$. Se observó EP en 20,8\% de los casos, de localización 


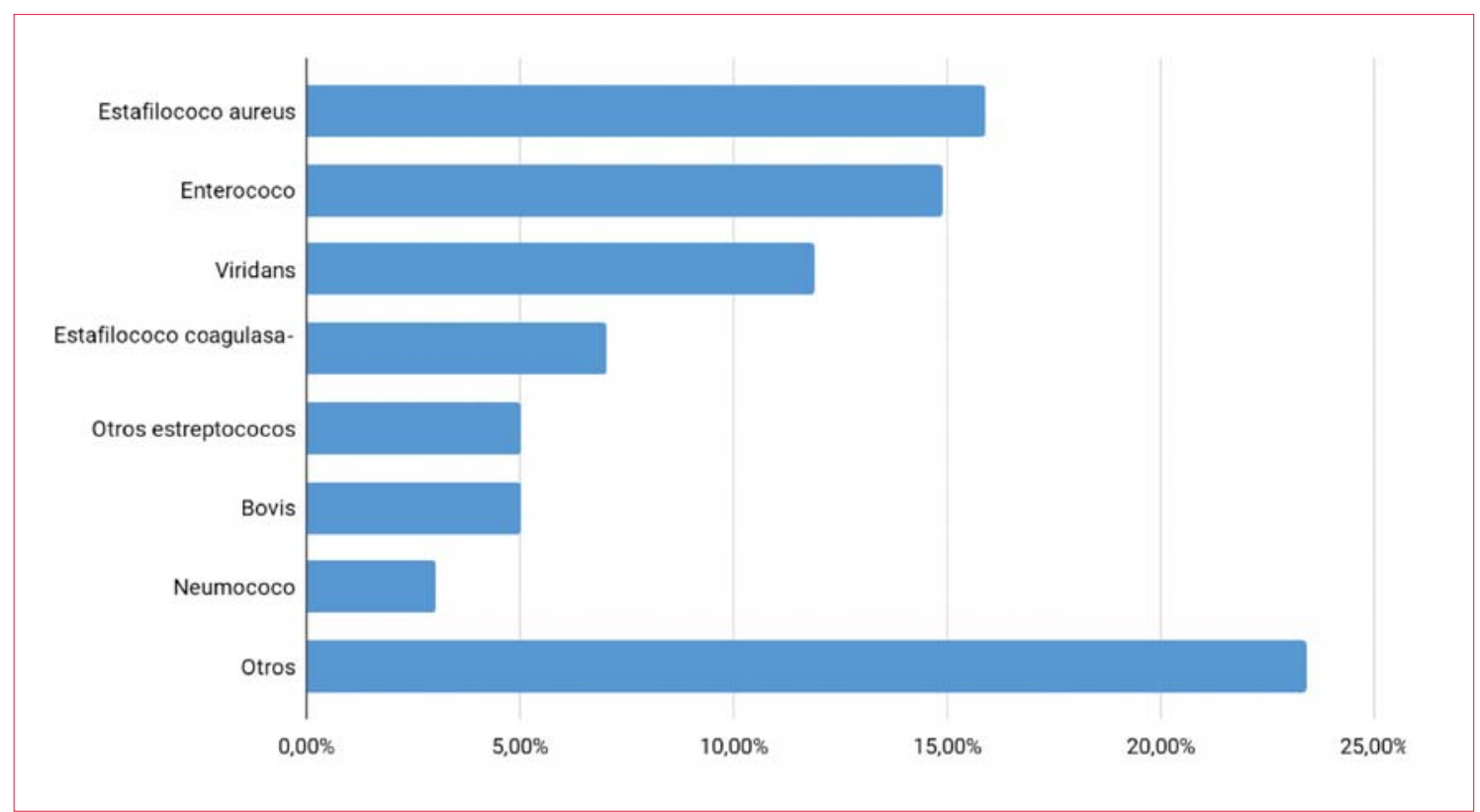

Figura 1. Microorganismos identificados.

Tabla 1. Características basales de la población.

\begin{tabular}{lc}
\hline Variable & Media $\pm D E$ \\
\hline Edad (años) & $57,9 \pm 15,4$ \\
Sexo masculino (n/\%) & $78(77,2)$ \\
Diabetes (n/\%) & $20(19,8)$ \\
HTA (n/\%) & $43(42,6)$ \\
Tabaquismo (n/\%) & $25(24,8)$ \\
Insuficiencia renal crónica (n/\%) & $5(5,0)$ \\
Sin cardiopatía congénita (n/\%) & $87(86,1)$ \\
VAB (n/\%) & $8(7,9)$ \\
Creatininemia (mg/dl) & $1,1 \pm 1,0$ \\
FEVI (\%) & $58,5 \pm 10,6$ \\
Puerta de entrada identificada (n/\%) & $37(36,6)$ \\
Fiebre al momento de cirugía (n/\%) & $18(17,8)$ \\
Tratamiento ATB previo (días) & $19,3 \pm 13,5$ \\
EI protésica precoz (n/\%) & $3(3,0)$ \\
EI protésica tardía (n/\%) & $4(4,0)$ \\
Procedimiento invasivo seis meses \\
previos (n/\%)
\end{tabular}

aórtica en 46,5\%, mitral 23,8\% y mitro-aórtica en $13,9 \%$. La mayoría de las intervenciones se realizaron en condición de urgencia-emergencia $(55,4 \%$ de los casos) (figura 3).

La MO global fue de $19,8 \%$, siendo mayor en pacientes con CL ( $29,2 \%$ vs $11,3 \%$ sin CL, p=0,025), única variable asociada a MO luego del análisis multivariado ( $\mathrm{OR}=3,38$; IC95\%: 1,0-11,3) (tabla 2). La incidencia de CL fue de 47,5\%, siendo el absceso la más frecuente (25,7\%). La EP (OR=5,8; IC95\%: 1,4-24,3), endocarditis sobre válvula aórtica $(\mathrm{OR}=2,9$; IC95\%: $1,1-7,8)$ y sexo masculino (OR=3,5; IC95\%: $1,1-11,4)$ se asociaron a CL tras el análisis multivariado (tabla 3).

La incidencia de embolias fue $31,7 \%$, localizadas principalmente en el sistema nervioso central (figura 4). Se asociaron a mayor riesgo de embolia: sexo femenino, edad, antecedente de hipertensión arterial (HTA), identificación de SA en HC y presencia de vegetaciones sobre valva posterior de la válvula mitral, pero luego del análisis multivariado resultaron predictores: SA en HC (OR=4,6; IC95\%: 1,2-18,6), vegetación en el velo mitral posterior $(\mathrm{OR}=3,9 ; \mathrm{IC} 95 \%$ : 1,1-10,7) y antecedente de HTA (OR=3,32; IC95\%: 1,3-8,4) (tabla 4). La incidencia de EP precoz fue de $3 \%$, y tardía de $4 \%$. De los siete pacientes con EP en la evolución, seis tuvieron CL en la presentación inicial $(p<0,05)$. Debido a la baja incidencia de EP en la evolución, no se lograron identificar predictores independientes.

La sobrevida global a cinco y diez años fue de $88,20 \%$ y $81,50 \%$ respectivamente (figura 5 ). La sobrevida fue menor en pacientes con CL y vegetación en el velo posterior (figura 6). Luego de la regresión de 


\begin{tabular}{|lcccc}
\hline \multicolumn{2}{l}{ Tabla 2. Predictores de mortalidad operatoria. } \\
\hline \multicolumn{2}{c}{ Variable } & \multicolumn{2}{c}{ Univariado } & Multivariado \\
\cline { 2 - 5 } & OR $(95 \%$ IC) & $p$ & OR $(95 \%$ IC) & $p$ \\
\hline Días ATB previo & $0,97(0,9-1,0)$ & 0,123 & - & - \\
SA & $2,38(0,7-7,9)$ & 0,163 & $3,6(1,1-9,5)$ & 0,13 \\
FEVI & $0,96(0,9-1,0)$ & 0,057 & $0,95(0,9-1,0)$ & 0,08 \\
CL & $3,2(1,1-9,5)$ & 0,029 & $3,38(1,1-11,3)$ & 0,04 \\
\hline
\end{tabular}

ATB: antibióticos; CL: complicaciones locales; FEVI: fracción de eyección del ventrículo izquierdo; SA: Staphylococcus aureus.

\begin{tabular}{|c|c|c|c|c|}
\hline \multirow[t]{2}{*}{ Variable } & \multicolumn{2}{|c|}{ Univariado } & \multicolumn{2}{|c|}{ Multivariado } \\
\hline & OR $(95 \%$ IC) & $p$ & OR $(95 \%$ IC) & $p$ \\
\hline Vegetación VAo & $1,96(0,87-4,45)$ & 0,10 & $2,9(1,1-7,8)$ & 0,049 \\
\hline Vegetación VP & $3,64(1,07-12,35)$ & 0,038 & $5,8(1,4-24,3)$ & 0,016 \\
\hline Sexo masculino & $4,10(1,35-12,42)$ & 0,031 & $3,5(1,1-11,4)$ & 0,037 \\
\hline
\end{tabular}

VAo: válvula aórtica; VP: válvula protésica.

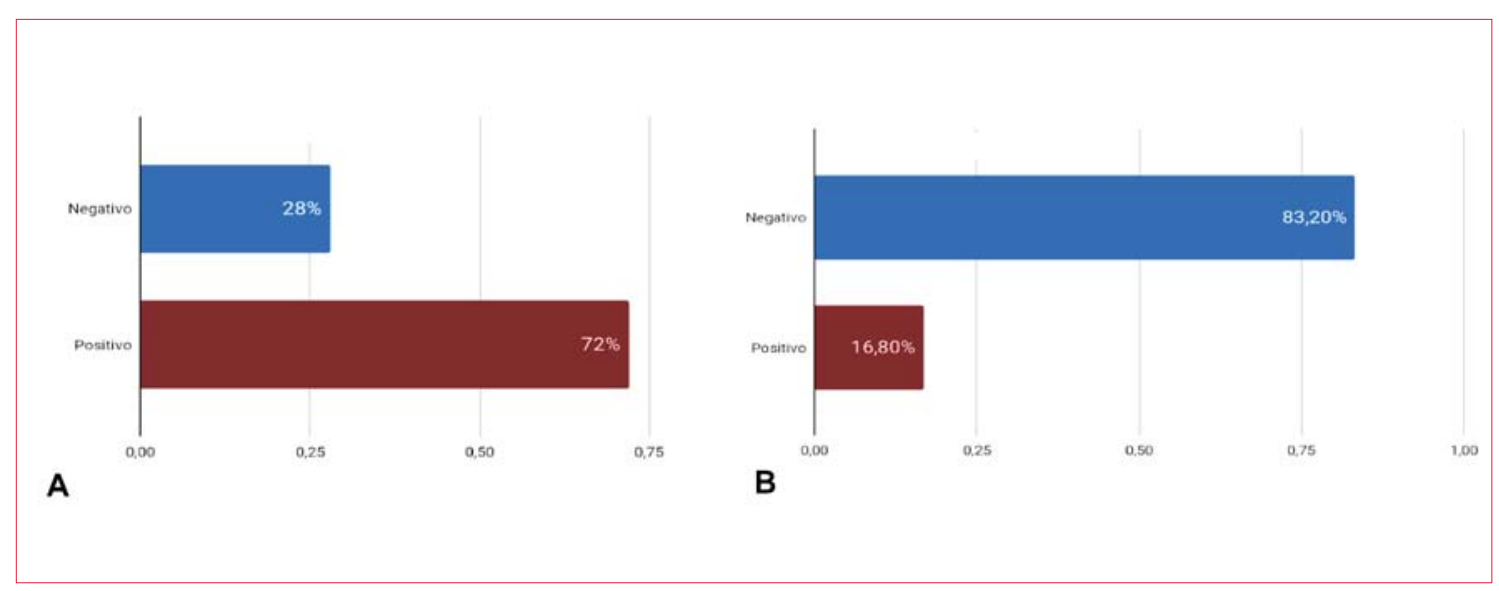

Figura 2. Porcentaje de pacientes con hemocultivos (A) y cultivos valvulares (B), positivos y negativos.

Cox, la presencia de CL (HR=3,1; IC95\%: 1,2-8,1) y vegetación en el velo posterior $(\mathrm{HR}=2,8$; IC95\%: 1,0-7,6) resultaron predictores de menor sobrevida. No fueron predictores de eventos adversos, ni la duración del tratamiento ATB previo a la cirugía, ni la presencia de fiebre al momento de la misma.

\section{Discusión}

En 1994, Durack y colaboradores ${ }^{(12)}$, de la Universidad de Duke, propusieron un esquema de diagnósti- co que estratificó a los pacientes con sospecha de EI en tres categorías: definida, posible y rechazada. Estos criterios han sido modificados en base a los avances en los métodos diagnósticos y al mayor conocimiento de la enfermedad ${ }^{(13)}$. El diagnóstico de EI debe realizarse precozmente para iniciar tratamiento ATB empírico inmediato e identificar a los pacientes con alto riesgo de complicaciones que pueden requerir un abordaje quirúrgico temprano.

La información respecto a la evolución de los pacientes operados por EI en nuestro medio es limita- 


\begin{tabular}{|c|c|c|c|c|}
\hline \multirow[t]{2}{*}{ Variable } & \multicolumn{2}{|c|}{ Univariado } & \multicolumn{2}{|c|}{ Multivariado } \\
\hline & OR $(95 \%$ IC) & $p$ & OR $(95 \%$ IC) & $p$ \\
\hline Sexo femenino & $2,42(0,9-6,5)$ & 0,079 & $2,5(0,8-7,6)$ & 0,12 \\
\hline Edad & $1,03(0,9-1,1)$ & 0,092 & $1,0(0,9-1,1)$ & 0,81 \\
\hline HTA & $3,1(1,2-8,1)$ & 0,019 & $3,32(1,3-8,4)$ & 0,023 \\
\hline SA & $2,94(0,9-8,0)$ & 0,058 & $4,6(1,2-18,6)$ & 0,021 \\
\hline Vegetación VPM & $2,68(0,9-7,3)$ & 0,054 & $3,9(1,1-10,7)$ & 0,019 \\
\hline
\end{tabular}

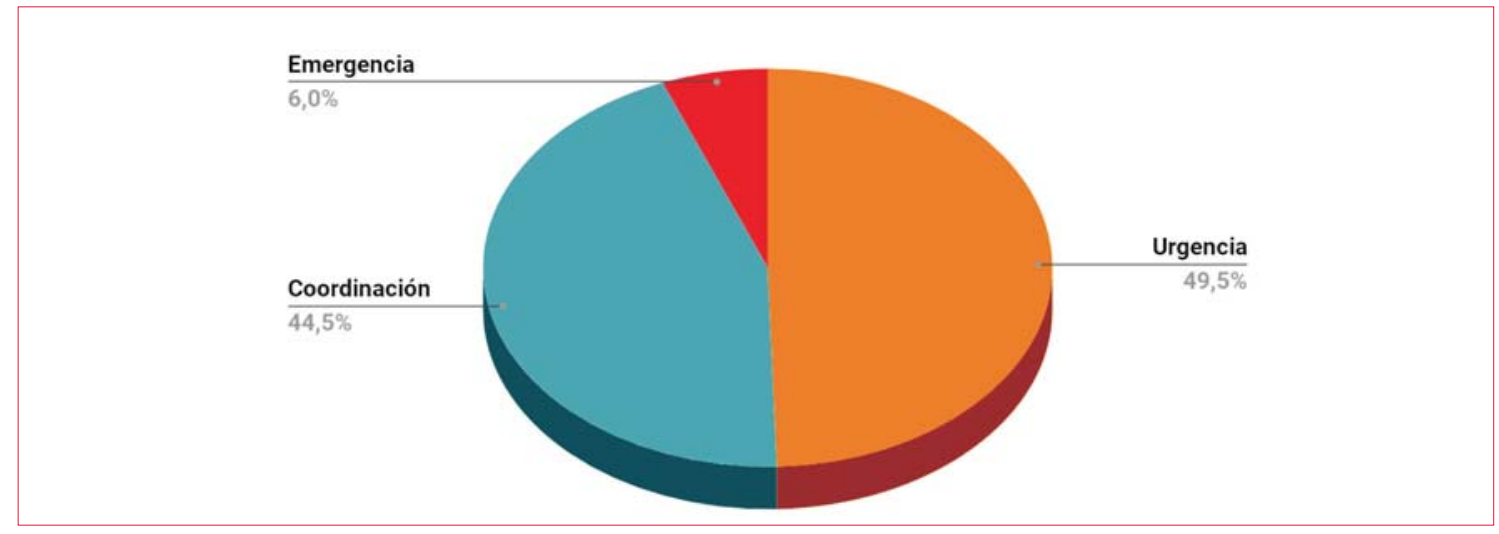

Figura 3. Oportunidad quirúrgica.

da. Por este motivo, y debido a la alta mortalidad y al perfil cambiante de la enfermedad, es fundamental conocer su estado actual.

En nuestra población observamos una edad media de 57,9 $( \pm 15,4)$ años, similar a la observada en el registro internacional ICE-PCS ${ }^{(4)}$ que incluyó 2.781 pacientes de 25 centros entre 2000 y 2005 . Está bien establecido el predominio de sexo masculino en la EI, como se reporta en este trabajo (72,9\%). Esto podría explicarse por la protección del endotelio mediada por estrógenos en la mujer en edad reproductiva(14) y por el desarrollo más tardío de enfermedad cardiovascular respecto a los hombres, lo que incluye a la enfermedad degenerativa valvular.

La presencia de condiciones predisponentes fue relativamente frecuente, principalmente debido a la presencia de válvula protésica (VP) (20,8\%) o cardiopatía congénita (13,9\%). A diferencia de estudios realizados hace cinco décadas en los que 50\% de los casos eran portadores de enfermedad mitral reumática(16), en nuestra población fueron tan solo $2 \%$.

Un grupo emergente en riesgo de EI está formado por pacientes con infecciones asociadas a los cuidados sanitarios ${ }^{(4)}$. Casi un tercio de los pacientes adquirieron EI como consecuencia de un procedimiento médico invasivo, al igual que lo observado en el EIRA 3, y otros registros internacionales ${ }^{(4)}$; esto evidencia la importancia de la profilaxis en este escenario, ya que es una infección potencialmente prevenible.

La microbiología de la enfermedad también se ha visto modificada. La incidencia de la EI causada por SA ha aumentado, siendo actualmente el agente etiológico más común en la mayor parte del mundo industrializado, incluyendo el registro argentino EIRA $3^{(5)}$. La aparición de EI por SA se vincula a los cuidados en salud (contacto hospitalario, procedimientos y dispositivos invasivos) como principal factor de riesgo, además del uso de drogas intravenosas. En contraste con los estreptococos, el SA no requiere la presencia de enfermedad valvular preexistente para determinar la infección ${ }^{(17)}$. La importancia de este patógeno potencialmente letal es motivo de preocupación dada la creciente resistencia antimicrobiana $^{(18)}$, incluso a la vancomicina ${ }^{(19)}$. En nuestro estudio, SA fue también el principal microorganismo causal (15,9\%). Siguieron en frecuencia: enterococo y viridans. El enterococo afecta habitualmente 


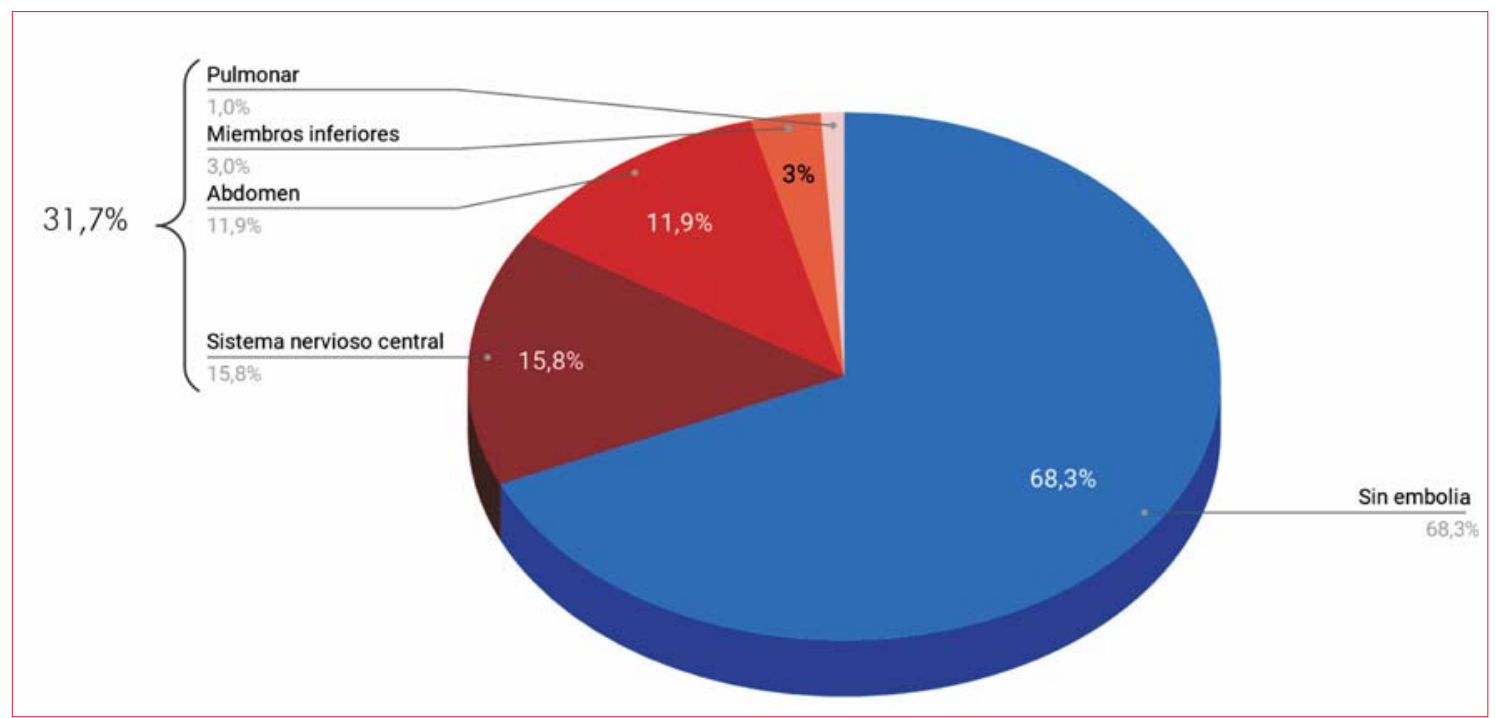

Figura 4. Localización de embolias.

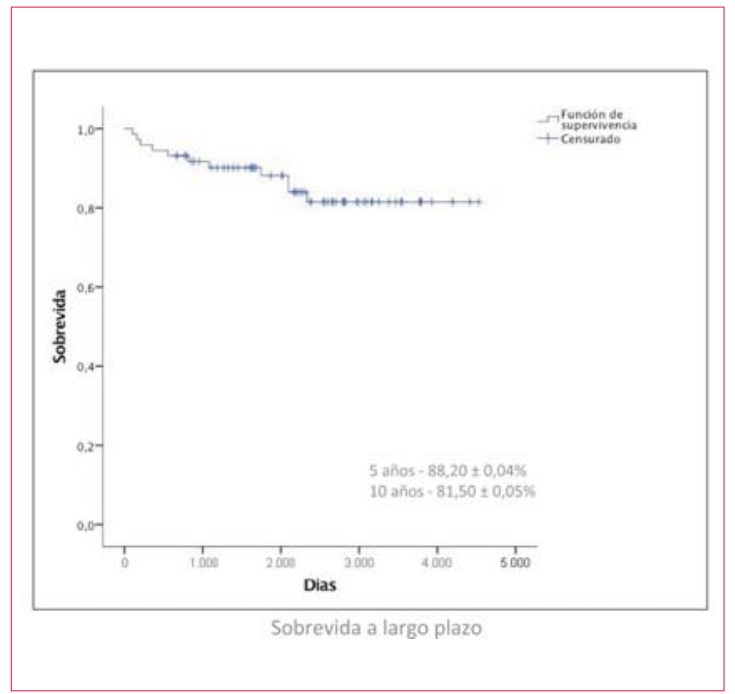

Figura 5. Sobrevida a largo plazo.

a pacientes ancianos con comorbilidades, y es más frecuente la adquisición nosocomial, la cual se asocia a peores resultados. La tasa de mortalidad reportada por este agente es intermedia entre la de estreptococos y la de estafilococos ${ }^{(20)}$.

El hecho de que casi un tercio de las EI ocurrieron en pacientes sometidos a un procedimiento invasivo en los seis meses previos y que en igual proporción se identificaron microorganismos asociados a infecciones nosocomiales, tiene implicancias importantes para el diagnóstico y el tratamiento de la EI en nuestro medio. Los pacientes que han recibido un procedimiento invasivo representan un grupo de riesgo que requiere atención diagnóstica cuidadosa en presencia de fiebre o bacteriemia. Este ha- llazgo pone en tela de juicio la implementación de profilaxis antibiótica en pacientes en riesgo.

Los HC fueron negativos en el $28 \%$ de los casos, cifra significativamente más alta que la hallada en registros internacionales (11,1\% en ICE-PCS[4]). Si bien $72 \%$ de los casos tuvo HC positivo, solo 16,8\% de los cultivos valvulares desarrolló microorganismos. Esto está probablemente vinculado al largo período de tratamiento ATB previo a la cirugía (media 19,3 días). Es importante destacar que ni la duración del tratamiento ATB previo a la cirugía, ni la presencia de fiebre al momento de la misma, resultaron ser predictores independientes de eventos adversos.

Se observó 20,8\% de EI sobre VP, similar a lo observado por Hoen B y colaboradores $(16 \%)^{(9)}$, y en el Euro Heart Survey (26\%) (21). Dentro de las infecciones sobre válvula nativa, la principal localización fue sobre válvula aórtica (VAo) (46,5\%), seguida de la válvula mitral (VM) (23,8\%). Nuestros resultados son similares a lo observado en el EIRA 3 (VAo 46\% VM $33 \%$ - VP 20,7\%). En ICE-PCS se observó EI sobre VP en el $22,2 \%$, seguida por la infección sobre VM $(41 \%)$ y VAo (38\%). Se encontró una elevada incidencia de complicaciones de EI, como embolias o CL. El 31,7\% de pacientes sufrió embolias, siendo la principal localización el sistema nervioso central, similar a lo reportado en EIRA 3 y en ICE-PCS (32,1\% y $40 \%$, respectivamente).

Diversos parámetros han sido identificados como predictores de eventos embólicos, como ser el tamaño y la movilidad de las vegetaciones ${ }^{(30)}$, el aumento del tamaño de la vegetación durante el tratamiento ATB ${ }^{(22)}$, la identificación de SA ${ }^{(22)}$, antece- 


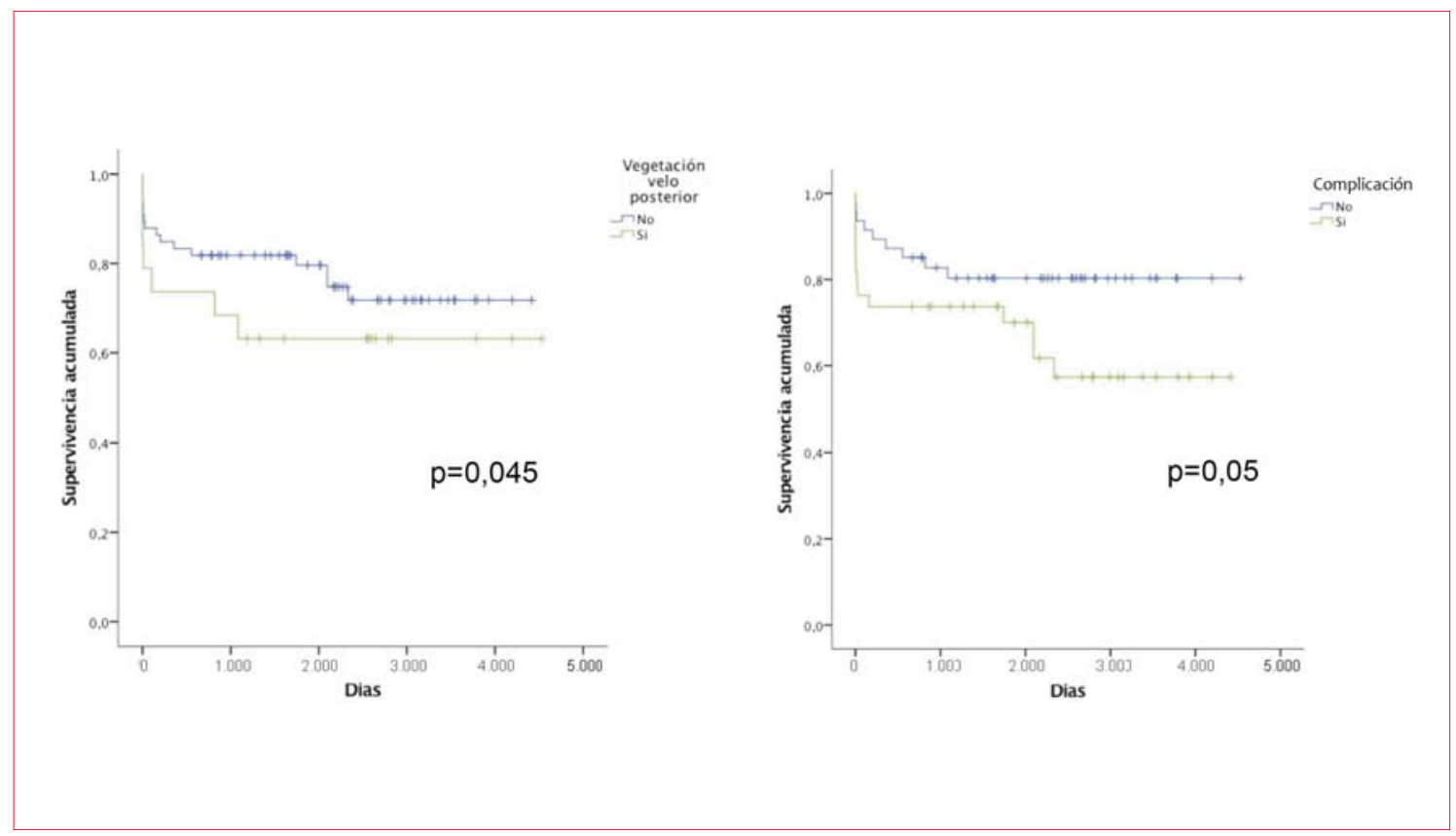

Figura 6. Sobrevida. Según la presencia de vegetación en el velo posterior de la válvula mitral (izquierda). Según la presencia de complicaciones locales (derecha).

dente de embolia previa ${ }^{(22)}$ y EI multivalvular. La localización de las vegetaciones sobre la VM ha generado controversias. Varios estudios han sugerido un mayor riesgo embólico en pacientes con endocarditis sobre VM. Pruitt y colaboradores informaron una tasa más alta de embolización cerebral y sistémica en pacientes con EI mitral en un estudio de 218 pacientes con $\mathrm{EI}^{(23)}$. Cabell y colaboradores encontraron en 145 pacientes con EI que la endocarditis mitral se asoció con un mayor riesgo de ataque cerebrovascular $(32,5 \% \text { vs } 11,3 \%, \mathrm{p}=0,003)^{(24)}$. En el estudio de Rohmann y colaboradores ${ }^{(25)}$, a 281 pacientes con sospecha clínica de EI se les practicó ecocardiograma transesofágico y se evaluó el valor pronóstico del sitio de la vegetación: las vegetaciones de la VM asociaron una incidencia significativamente mayor de eventos embólicos que las de la válvula aórtica ( $25 \%$ vs $9,7 \%$ ). Particularmente la valva anterior mitral ha sido identificada como la localización de mayor potencial embolígeno por algunos autores. Sin embargo, las vegetaciones en la VM fueron significativamente mayores que las de la VAo y se ha puesto en duda si la mayor incidencia de eventos embólicos en la endocarditis mitral podría deberse al mayor tamaño de la vegetación y no a su ubicación. Un estudio europeo multicéntrico no encontró diferencias en eventos embólicos entre EI de la VM y aórtica, que ocurrieron en 70 de 191 pacientes $(37 \%)$ sobre VM, y 67 de 214 pacientes (31\%) sobre $\mathrm{VAo}^{(26)}$. Un estudio reciente de 847 pacientes con EI encontró seis factores (edad, diabetes mellitus, fibrilación auricular, embolia previa, longitud de la vegetación e infección por SA) asociados a un aumento del riesgo embólico, que se utilizaron para crear una calculadora del riesgo embólico ${ }^{(27)}$.

En nuestro estudio los únicos predictores independientes de embolias fueron: identificación de SA en HC, vegetación en el velo mitral posterior y antecedente de HTA (tabla 4). Los efectos mecánicos de la excursión amplia y abrupta de las valvas mitrales, que ocurren dos veces por latido en ritmo sinusal, podrían contribuir a la fragmentación y embolización de las vegetaciones, determinando así mayor incidencia de eventos embólicos.

La incidencia de CL en registros internacionales se encuentra en torno a $37 \%{ }^{(5)}$ y el absceso perivalvular es la más habitual ${ }^{(28)}$. Es más frecuente en la EI aórtica (10\%-40\%) y en la EP (56\%-100\%) ${ }^{(28)}$. En nuestro grupo de pacientes intervenidos por EI, la incidencia de CL fue de 47,5\%, más frecuentemente el absceso (25,7\%). Los factores de riesgo de CL más importantes identificados en otros estudios fueron: EP, endocarditis sobre VAo e infección por estafilococo coagulasa negativo ${ }^{(28)}$. En nuestra población, encontramos coincidentemente que tanto la EP como la endocarditis aórtica, se vieron asociadas de forma independiente a CL, junto con el sexo masculino (tabla 3).

La MO global registrada en estudios internacionales es alta $(12 \%-22 \%)^{(5)}$. En nuestra población fue $19,8 \%$, siendo mayor en pacientes con CL (29,5\% vs $11,3 \%, \mathrm{p}=0,025)$. La única variable que vimos asociada a mayor MO, luego del análisis multivariado, fue la presencia de CL (OR=3,38; IC95\%: 1,1-11,3). 
Adicionalmente, encontramos que las CL aumentan el riesgo de EP en la evolución. En las series recientes, las tasas de supervivencia a largo plazo se encuentran en $60 \%-70 \%$ a los cinco años ${ }^{(29-31)}$. En diez años de seguimiento la supervivencia fue de $74,8 \%$ en el estudio de Pang PY y colaboradores ${ }^{(32)}$. En nuestra población, la sobrevida global a cinco y diez años fue mayor que la reportada, 88,20\% y $81,50 \%$, respectivamente (figura 5).

Ya fue demostrado que la presencia de CL es un predictor de mal pronóstico a largo plazo ${ }^{(33)}$. Otros factores identificados como predictores de sobrevida a largo plazo son: edad, EP, infección por SA y fracción de eyección del ventrículo izquierdo $\leq 45 \%{ }^{(32)}$. La sobrevida a largo plazo en nuestra población fue menor en pacientes con CL y con vegetación en el velo posterior mitral (figura 6). Como se observa en la figura 6, la sobrevida de pacientes con CL es peor que en pacientes sin CL, lo que podría explicarse por la mayor incidencia de EP. Resulta novedoso que los pacientes con localización en el velo posterior mitral hayan tenido peor evolución. En vista de este peor resultado, estos pacientes deberían ser seguidos estrechamente, incluso a largo plazo, a pesar de tener una intervención quirúrgica temprana.

\section{Limitaciones}

Se trata de un estudio observacional retrospectivo con sesgos propios de la selección de pacientes candidatos a cirugía. Se realizó en un solo centro quirúrgico, lo que probablemente limite la aplicación de los hallazgos a otros entornos. Una serie prospectiva más amplia y un enfoque multicéntrico nacional, permitirían obtener nuevas conclusiones respecto a los factores pronósticos identificados. El bajo número de EP en la evolución ha limitado el uso del análisis multivariado para evaluar los factores de riesgo.

\section{Conclusiones}

La MO de la EA que recibió cirugía cardíaca en nuestro medio es similar a la reportada internacionalmente. Las CL se asocian a EP en la evolución y resultaron ser un predictor independiente de sobrevida a largo plazo. La presencia de vegetaciones sobre el velo posterior mitral, el antecedente de HTA y la infección a SA fueron predictores independientes de embolias. La presencia de CL resultó ser el principal predictor de muerte. La sobrevida a largo plazo de pacientes operados en nuestro medio es muy buena.

\section{Contribución de autores}

\section{Diego Pérez Zerpa,}

https://orcid.org/0000-0002-6919-6545, contribución a la concepción y diseño del estudio, adquisición de datos, análisis e interpretación de los datos, redacción del trabajo y aprobación definitiva de la versión que se presenta.

\section{Amparo Fernández,}

https://orcid.org/0000-0003-4243-7844, contribución a la concepción y diseño del estudio, adquisición de datos, análisis e interpretación de los datos, redacción del trabajo y aprobación definitiva de la versión que se presenta.

Facundo Ríos, https://orcid.org/0000-0002-6868-3480, contribución a la concepción y diseño del estudio, adquisición de datos, análisis e interpretación de los datos, redacción del trabajo y aprobación definitiva de la versión que se presenta.

Eloísa Silva, https://orcid.org/0000-0001-5595-1792, contribución al análisis e interpretación de los datos, redacción del trabajo y aprobación definitiva de la versión que se presenta.

\section{Macarena Lorente,}

https://orcid.org/0000-0003-2974-6028, contribución al análisis e interpretación de los datos, redacción del trabajo y aprobación definitiva de la versión que se presenta.

María José Arocena,

http://orcid.org/0000-0003-4072-7426, contribución al análisis e interpretación de los datos, redacción del trabajo y aprobación definitiva de la versión que se presenta.

\section{Jorge Estigarribia,}

https://orcid.org/0000-0002-5136-7668, contribución a la concepción y diseño del estudio, interpretación de los datos, revisión crítica y aprobación definitiva de la versión que se presenta.

Gerardo Soca, https://orcid.org/0000-0002-8073-3292, contribución a la concepción y diseño del estudio, interpretación de los datos, revisión crítica del contenido intelectual y aprobación definitiva de la versión que se presenta.

Víctor Dayan, https://orcid.org/0000-0002-5470-0585, contribución a la concepción y diseño del estudio, interpretación de los datos, revisión crítica y aprobación definitiva de la versión que se presenta.

Este artículo fue aceptado para su publicación por: Editor jefe Dr. Gerardo Soca.

\section{Bibliografía}

1. Duval X, Delahaye F, Alla F, Tattevin P, Obadia JF, Le Moing V, et al. Temporal trends in infective endocarditis in the context of prophylaxis guideline modifications: three successive population-based surveys. J Am Coll Cardiol. 2012;59(22):1968-76. doi:10.1016/j.jacc.2012.02.029.

2. Correa de Sa DD, Tleyjeh IM, Anavekar NS, Schultz JC, Thomas JM, Lahr BD, et al. Epidemiological trends of infective endocarditis: a population-based study in Olmsted County, Minnesota. Mayo Clin Proc. 2010;85(5):422-6. doi:10.4065/mcp.2009.0585 
3. Toyoda N, Chikwe J, Itagaki S, Gelijns AC, Adams DH, Egorova NN. Trends in infective endocarditis in California and New York State, 1998-2013. JAMA. 2017;317(16):1652-60. doi: 10.1001/jama. 2017.4287

4. Murdoch DR, Corey GR, Hoen B, Miro JM, Fowler VG, Jr., Bayer AS, et al. Clinical presentation, etiology, and outcome of infective endocarditis in the 21st century: the International Collaboration on Endocarditis-Prospective Cohort Study. Arch Intern Med 2009;169:463-73. doi: 10.1001/archinternmed.2008.603.

5. Avellana PM, García Aurelio M, Swieszkowski S, Nacinovich F, Kaselian L, Spennato M, et al. Endocarditis infecciosa en la República Argentina. Resultados del estudio EIRA 3. Rev Argent Cardiolog. 2018; 86(1):20-1. doi: 10.7775/rac.es.v86.i1.10935.

6. Casabe JH, Hershson A, Ramos MS, Barisani JL, Pellegrini C, Varini S. Endocarditis infecciosa en la República Argentina. Complicaciones y Mortalidad. Rev Argent Cardiol. 1996;64:39-45.

7. Ferreirós E, Nacinovich F, Casabe JH, Modenesi JC, Swieszkowski S, Cortes C, et al. Epidemiologic, clinical, and microbiologic profile of infective endocarditis in Argentina: a national survey. The Endocarditis Infecciosa en la Republica Argentina-2 (EIRA-2) Study. Am Heart J 2006;151(2):545-52. doi: 10.1016/j.ahj.2005.04.008

8. Tleyjeh IM, Abdel-Latif A, Rahbi H, Scott CG, Bailey KR, Steckelberg JM, et al. A systematic review of population-based studies of infective endocarditis.Chest. 2007; 132(3):1025-35. doi: 10.1378/chest. 06-2048

9. Hoen B, Alla F, Selton-Suty C, Beguinot I, Bouvet $A$, Briancon $S$, et al. Changing profile of infective endocarditis: results of a 1-year survey in France. JAMA. 2002;288(1):75-81. doi: 10.1001/jama.288. 1.75

10. Castillo JC, Anguita MP, Ruiz M, Peña L, Santisteban M, Puentes M, et al. Cambios epidemiológicos de la endocarditis infecciosa sobre válvula nativa. Rev Esp Cardiol. 2011;64(7):594-8. doi:10 1016/j.recesp.2011.03.011

11. Habib G, Lancellotti P, Antunes MJ, Bongiorni MG, Casalta J-P, Del Zotti F, et al. Guías ESC 2015 sobre el tratamiento de la endocarditis infecciosa. Rev Esp Cardiol. 2016;69(1):e1-49. doi: 10.1016 j.recesp.2015.11.015

12. Durack, DT, Lukes AS, Brigth DK. New criteria for diagnosis of infective endocarditis: utilization of specific echocardiographic findings. Duke Endocarditis Service. Am J Med.1994;96(3):200-9. doi:10.1016/0002-9343(94)90143-0

13. Li JS, Sexton DJ, Mick N, Nettles R, Fowler VG, Ryan T, et al. Proposed modifications to the Duke criteria for the diagnosis of infective endocardi- tis. Clin Infect Dis. 2000;30(4):633-8. doi: 10.1086/ 313753.

14. Bakir S, Mori T, Durand J, Chen YF, Thompson JA, Oparil S. Estrogen-induced vasoprotection is estrogen receptor dependent: evidence from the balloon-injured rat carotid artery model. Circulation 2000; 101(20): 2342-4. doi:10.1161/01.cir.101.20.2342

15. Pelletier LL, Petersdorf RG. Infective endocarditis: a review of 125 cases from the University of Washington Hospitals, 1963-72. Medicine (Baltimore) 1977;56(4):287-313.

16. Weinstein L, Rubin RH. Infective endocarditis-1973. Prog Cardiovasc Dis. 1973;16(3) 239-74. doi: 10.1016/s0033-0620(73)80001-5.

17. Moreillon P, Que YA. Infective endocarditis. Lancet 2004;363(9403):139-49. doi:10.1016/S0140-6736 (03)15266-X

18. Naimi TS, LeDell KH, Como-Sabetti K, Borchardt SM, Boxrud DJ, Etienne J, et al. Comparison of community- and health care-associated methicillin-resistant Staphylococcus aureus infection. JAMA 2003;290(22):2976-84. doi:10.1001/jama.290. 22.2976 .

19. Whitener CJ, Park SY, Browne FA, Parent LJ, Julian K, Bozdogan B, et al. Vancomycin-resistant Staphylococcus aureus in the absence of vancomycin exposure. Clin Infect Dis. 2004;38(8):104955. doi:10.1086/382357.

20. Chirouze C, Athan E, Alla F, Chu VH, Ralph Corey G, Selton-Suty $\mathbf{C}$, et al. Enterococcal endocarditis in the beginning of the 21st century: analysis from the International Collaboration on Endocarditis-Prospective Cohort Study. Clin Microbiol Infect. 2013;19(12):1140-7. doi: 10.1111/1469-0691.12166.

21. Tornos P, Iung B, Permanyer-Miralda G, Baron G, Delahaye F, Gohlke-Bärwolf $\mathrm{Ch}$, et al. Infective endocarditis in Europe: lessons from the Euro heart survey. Heart 2005;91(5):571-5. doi:10.1136/hrt.2003.032128.

22. Vilacosta I, Graupner C, San Román JA, Sarriá C, Ronderos R, Fernández C, et al. Risk of embolization after institution of antibiotic therapy for infective endocarditis. J Am Coll Cardiol. 2002;39(9):1489-95. doi:10.1016/s0735-1097(02)01790-4.

23. Pruitt AA, Rubin RH, Karchmer AW, Duncan GW. Neurologic complications of bacterial endocarditis. Medicine (Baltimore). 1978;57(4):329-43. doi: 10.1097/00005792-197807000-00004

24. Cabell CH, Pond KK, Peterson GE, Durack DT, Corey GR, Anderson DJ, et al. The risk of stroke and death in patients with aortic and mitral valve endocarditis. Am Heart J. 2001;142(1):75-80. doi:10.1067/mhj.2001.115790.

25. Rohmann S, Erbel R, Gorge G, Makowski T, Mohr-Kahaly S, Nixdorff U, Drexler M, Meyer 
J. Clinical relevance of vegetation localization by transoesophageal echocardiography in infective endocarditis. Eur Heart J. 1992;13:446-52. doi: 10.1093/ oxfordjournals.eurheartj.a060195

26. Thuny F, Di Salvo G, Disalvo G, Belliard O, Avierinos JF, Pergola V, et al. Risk of embolism and death in infective endocarditis: prognostic value of echocardiography: a prospective multicenter study. Circulation 2005;112(1):69-75. doi:10.1161/ CIRCULATIONAHA.104.493155.

27. Hubert S, Thuny F, Resseguier N, Giorgi R, Tribouilloy C, Le Dolley Y, et al. Prediction of symptomatic embolism in infective endocarditis: construction and validation of a risk calculator in a multicenter cohort. J Am Coll Cardiol. 2013; 62:1384-92. doi: 10.1016/j.jacc.2013.07.029.

28. Habib G, Lancelloti P, Antunes MJ, Bongiorni MG, Casalta JP, Del Zotti F, et al. 2015 ESC guidelines for management of infective endocarditis: the task forces for the management of infective endocarditis for the European Society of Cardiology. (ESC). Endorsed by: European Association for Cardio-Thoracic Surgery ((EACTS), the European Association of Nuclear Medicine (EANM). Eur Heart J. 2015;36(44): 3075-3128. doi:10.1093/eurheartj/ehv319.

29. Thuny F, Giorgi R, Habachi R, Ansaldi S, Le Dolley Y, Casalta JP, et al. Excess mortality and morbidity in patients surviving infective endocarditis. Am Heart J. 2012;164(1):94-101.

doi:10.1016/j.ahj.2012.04.003.

30. Fernández-Hidalgo N, Almirante B, Tornos $\mathbf{P}$, González-Alujas MT, Planes AM, Galiñanes M, et al. Immediate and long-term outcome of left-sided infective endocarditis. A 12-year prospective study from a contemporary cohort in a referral hospital. Clin Microbiol Infect. 2012;18(12):E522-30. doi:10. 1111/1469-0691.12033.

31. Mokhles MM, Ciampichetti I, Head SJ, Takkenberg JJ, Bogers AJ. Survival of surgically treated infective endocarditis: a comparison with the general dutch population. Ann Thorac Surg. 2011;91(5):1407-12. doi:10.1016/j.athoracsur.2011. 02.007 .

32. Pang PY, Sin YK, Lim CH, Tan TE, Lim SL, Chao VT, et al. Surgical management of infective endocarditis: an analysis of early and late outcomes. Eur J Cardiothorac Surg. 2015;47(5):826-32. doi:10.1093/ejcts/ezu281.

33. Chan KL. Early clinical course and long-term outcome of patients with infective endocarditis complicated by perivalvular abscess. CMAJ. 2002;167(1): 19-24. 